\title{
Diffuse Alveolar Hemorrhage Associated with Dilated Cardiomyopathy and Sleep Apnea Syndrome
}

\author{
Ryohei Suematsu ${ }^{1}$, Jun Miyata ${ }^{1}$, Tomoya Sano ${ }^{1}$, Chie Watanabe ${ }^{1}$, Yohei Maki ${ }^{1}$, \\ Yoshifumi Kimizuka ${ }^{1}$, Nobuyoshi Hayashi ${ }^{1}$, Yuji Fujikura ${ }^{1}$, Hiroaki Sugiura ${ }^{2}$, \\ Hiroshi Shinmoto ${ }^{2}$, Akira Taruoka ${ }^{3}$, Yuji Nagatomo ${ }^{3}$, Takeshi Adachi ${ }^{3}$ and Akihiko Kawana ${ }^{1}$
}

\begin{abstract}
:
We herein report a case of diffuse alveolar hemorrhage (DAH) associated with dilated cardiomyopathy (DCM) and sleep apnea syndrome (SAS) in a 47-year-old man. The patient exhibited recurring dyspnea and bloody sputum. Chest radiography showed bilateral diffuse infiltrative opacities without pleural effusion. A bronchoscopic analysis of bronchoalveolar lavage fluid revealed hemosiderin-laden macrophages. Based on these findings, he was diagnosed with DAH. Laboratory and pathological findings ruled out the possibility of collagen diseases and vasculitis. Overnight polysomnography revealed concomitant severe obstructive SAS. Treatment with continuous positive-pressure ventilation and pharmacological therapy for DCM prevented recurrence of DAH.
\end{abstract}

Key words: diffuse alveolar hemorrhage, dilated cardiomyopathy, anemia, sleep apnea syndrome, negative pressure pulmonary hemorrhage

(Intern Med 60: 1911-1914, 2021)

(DOI: 10.2169/internalmedicine.5219-20)

\section{Introduction}

Diffuse alveolar hemorrhage (DAH) is a persistent and recurrent syndrome caused by rupture of the pulmonary microvasculature (1) and typically presents with dyspnea, bloody sputum, and/or hemoptysis (2). Anemia is a key laboratory finding suggestive of this disease. Previous studies have reported that $25-50 \%$ of patients with DAH die due to rapidly progressive respiratory failure (3). DAH can develop secondary to collagen diseases, including systemic lupus erythematosus, vasculitis (4), or adverse events related to pharmacological agents, such as anticoagulants and antiplatelet drugs. Other rare diseases involving antiphospholipid syndrome and idiopathic pulmonary hemosiderosis have also been reported (5-7). Most cases of idiopathic DAH with an undetermined pathophysiology are difficult to treat.
Dilated cardiomyopathy (DCM) is characterized by decreased left ventricular contraction and dilation of the left ventricular cavity, which precipitate a reduced cardiac output leading to heart failure. There is no radical treatment available at present other than heart transplantation. DCM comprises idiopathic as well as secondary types, including sarcoidosis and amyloidosis, which produce systemic manifestations.

Patients with sleep apnea syndrome (SAS) experience repeated apnea and hypopnea during sleep. Upper airway obstruction, often caused by obesity or hypertrophic pharyngeal tonsils, frequently occurs in patients with SAS. These phenomena induce hypoxemia and increase blood pressure, which can lead to various complications.

To our knowledge, this is the first report of a patient with DAH accompanied by SAS and/or DCM. In addition, we also discuss the pathophysiological mechanism underlying these rare complications.

\footnotetext{
${ }^{1}$ Division of Infectious Diseases and Respiratory Medicine, Department of Internal Medicine, National Defense Medical College, Japan, ${ }^{2}$ Department of Radiology, National Defense Medical College, Japan and ${ }^{3}$ Division of Cardiovascular Medicine, Department of Internal Medicine, National Defense Medical College, Japan

Received: April 30, 2020; Accepted: December 6, 2020; Advance Publication by J-STAGE: February 1, 2021

Correspondence to Dr. Jun Miyata, junmiyata@ndmc.ac.jp
} 
Table. Results of Blood Test on Admission.

\begin{tabular}{|c|c|c|c|c|c|}
\hline \multicolumn{2}{|l|}{ Hematology } & \multicolumn{2}{|c|}{ Biochemistry } & \multicolumn{2}{|l|}{ Serology } \\
\hline White blood cells & $5,000 / \mathrm{uL}$ & T-Bil & $0.71 \mathrm{mg} / \mathrm{dL}$ & CRP & $27 \mathrm{mg} / \mathrm{dL}$ \\
\hline Neutrophil & $80.4 \%$ & AST & 72 IU/L & BNP & $232 \mathrm{pg} / \mathrm{mL}$ \\
\hline Lymphocyte & $16.6 \%$ & ALT & $31 \mathrm{IU} / \mathrm{L}$ & KL-6 & $389 \mathrm{U} / \mathrm{mL}$ \\
\hline Basophil & $0.2 \%$ & LDH & 777 IU/L & $\mathrm{ACE}$ & 8.7 IU/L \\
\hline Eosinophil & $0 \%$ & TP & $6.5 \mathrm{~g} / \mathrm{dL}$ & sIL-2R & $772 \mathrm{U} / \mathrm{mL}$ \\
\hline Monocyte & $2.8 \%$ & Alb & $3.3 \mathrm{~g} / \mathrm{dL}$ & $1-3-\beta$-D glucan & $13 \mathrm{pg} / \mathrm{mL}$ \\
\hline Red blood cells & $395 \times 10^{4} / \mu \mathrm{L}$ & BUN & $29 \mathrm{mg} / \mathrm{dL}$ & & \\
\hline Hemoglobin & $9.6 \mathrm{~g} / \mathrm{dL}$ & $\mathrm{Cr}$ & $1.37 \mathrm{mg} / \mathrm{dL}$ & & \\
\hline Hematocrit & $30.1 \%$ & $\mathrm{Na}$ & $135 \mathrm{mEq} / \mathrm{L}$ & & \\
\hline \multirow[t]{2}{*}{ Platelets } & $18 \times 10^{4} / \mu \mathrm{L}$ & K & $4.3 \mathrm{mEq} / \mathrm{L}$ & & \\
\hline & & $\mathrm{Cl}$ & $101 \mathrm{mEq} / \mathrm{L}$ & & \\
\hline
\end{tabular}

ACE: angiotensin-converting enzyme, BNP: brain natriuretic peptide, KL-6: Krebs von den Lungen-6, sIL-2R: soluble interleukin-2 receptor

\section{Case Report}

A 47-year-old obese man (height, $170 \mathrm{~cm}$; weight, $80 \mathrm{~kg}$; BMI, 27.7), who had been treated for hypertension with an angiotensin II receptor antagonist (irbesartan $100 \mathrm{mg} /$ day) and a calcium channel blocker (amlodipine $5 \mathrm{mg} / \mathrm{day}$ ), complained of dyspnea during exertion. His blood pressure was $118 / 68 \mathrm{mmHg}$ and well-controlled under treatment. Ultrasonic echocardiography revealed a decreased left ventricular contraction, dilated left ventricular cavity (end-diastolic diameter $63 \mathrm{~mm}$ ), and impaired ejection fraction (32\%). However, there was no significant stenosis in the coronary artery. Right heart catheterization showed a high pulmonary arterial pressure (mean $47 \mathrm{mmHg}$ ) and pulmonary capillary wedge pressure (mean $32 \mathrm{mmHg}$ ), suggestive of a left-side cardiac failure leading to post-capillary pulmonary arterial hypertension. However, the cardiac index was $2.96 \mathrm{~L} / \mathrm{min} / \mathrm{m}^{2}$, which was within the normal range.

A myocardial biopsy was performed, which showed no granulomas or amyloid deposits. ${ }^{18} \mathrm{~F}$-2-deoxy-2-fluoro-Dglucose (FDG) positron emission tomography (PET) revealed a low FDG uptake in the ventricular septum. Based on these findings, he was diagnosed with idiopathic DCM. Additional treatment with a selective aldosterone receptor antagonist (eplerenone $25 \mathrm{mg} /$ day) was initiated, and he was followed at an outpatient clinic.

Three months later, he presented with fever, bloody sputum, and worsened dyspnea. His blood pressure and peripheral oxygen saturation were $120 / 70 \mathrm{mmHg}$ and $77 \%$ on room air, respectively. He was not taking any antiplatelet drugs or anticoagulants. He had no history of vasculitis or autoimmune disorders involving joint pains, skin rash, photosensitivity, or Raynaud's phenomenon.

A laboratory examination demonstrated microcytic anemia (hemoglobin level, $9.6 \mathrm{~g} / \mathrm{dL}$; mean corpuscular volume, 76 $\mathrm{fL}$ ). Coagulation studies were normal, therefore ruling out thrombocytopenia. Serum markers including antinuclear antibody, anti-neutrophil cytoplasmic antibodies, anti- cardiolipin antibody, and anti-glomerular basement membrane antibody were negative. Serum levels of angiotensinconverting enzyme and lysozyme were within the normal range (Table). Ultrasonic echocardiography revealed a worsened ejection fraction (20\%). Chest radiography (Fig. 1A) and computed tomography $(\mathrm{CT})$ (Fig. 1B, C) revealed bilateral diffuse ground glass opacities (GGOs) with intact peripheral lesions; however, pleural effusion was not observed.

We administered antibiotic agents for possible bacterial pneumonia. However, a sputum examination did not demonstrate any bacterial pathogens. His symptoms and abnormal imaging findings gradually improved (Fig. 2A-C). However, he developed recurrence of bloody sputum one month later. Chest radiograph and $\mathrm{CT}$ showed consolidation surrounded by GGO in the upper right lobe (Fig. 3A-C). Bronchoscopy was performed, showing bloody effusion in the right upper bronchus (Fig. 4A). An analysis of the bronchoalveolar lavage fluid collected from the right middle lobe branch (Fig. 4B) revealed hemosiderin-laden macrophages (Fig. 4C). The proportions of lymphocytes and eosinophils were within a normal range. A histological analysis demonstrated no granulomas, amyloid or hemosiderin deposits, or vascular inflammation. Based on these findings, the patient was diagnosed with DAH.

Nocturnal hypoxia with apnea and hypopnea was observed by overnight pulse oximetry monitoring during hospitalization. He was overweight suggestive of concomitant SAS. Polysomnography was performed, which showed a high apnea-hypopnea index (4\% oxygen desaturation index: 62.0 times/h; minimum peripheral oxygen saturation: $72 \%$; obstructive apnea index: 6.4 times/h; central apnea index: 0 times/h; mixed apnea index: 0.6 times/h; hypopnea index: 48.7 times/h) with frequent upper airway obstruction. The patient was therefore diagnosed with severe SAS.

After treatment with an auto-adjusting continuous positive-pressure ventilation device (maximal positive airway pressure, $20 \mathrm{cmH}_{2} \mathrm{O}$; minimum positive airway pressure, 4 $\mathrm{cmH}_{2} \mathrm{O}$; mean positive airway pressure, $10 \mathrm{cmH}_{2} \mathrm{O}$ ), which improved the apnea-hypopnea index to 5 times/h, in addition 

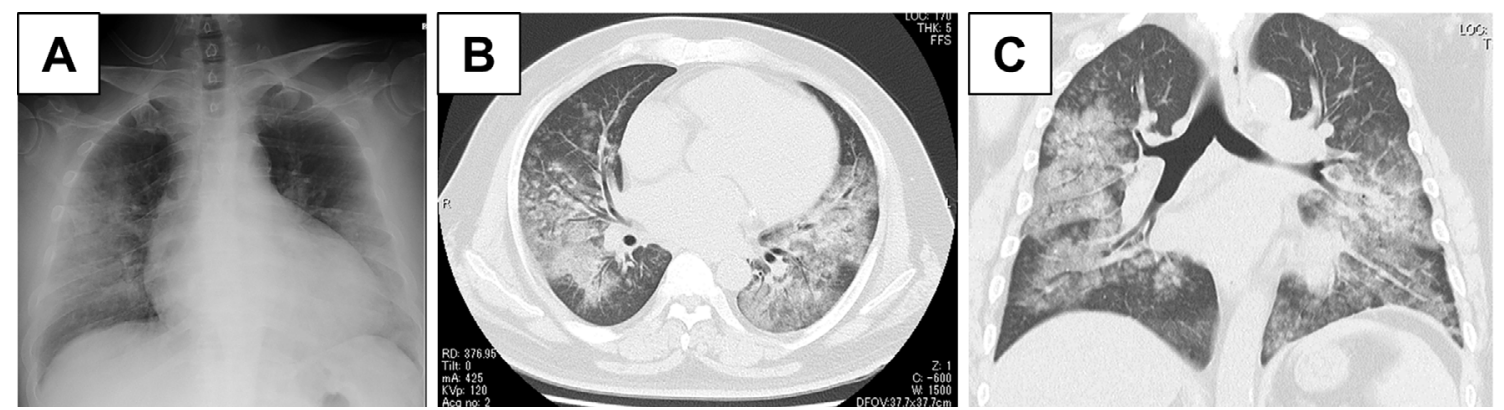

Figure 1. (A) Chest radiography showed bilateral infiltration, and (B, C) computed tomography showed bilateral diffuse ground glass opacity without pleural effusion.
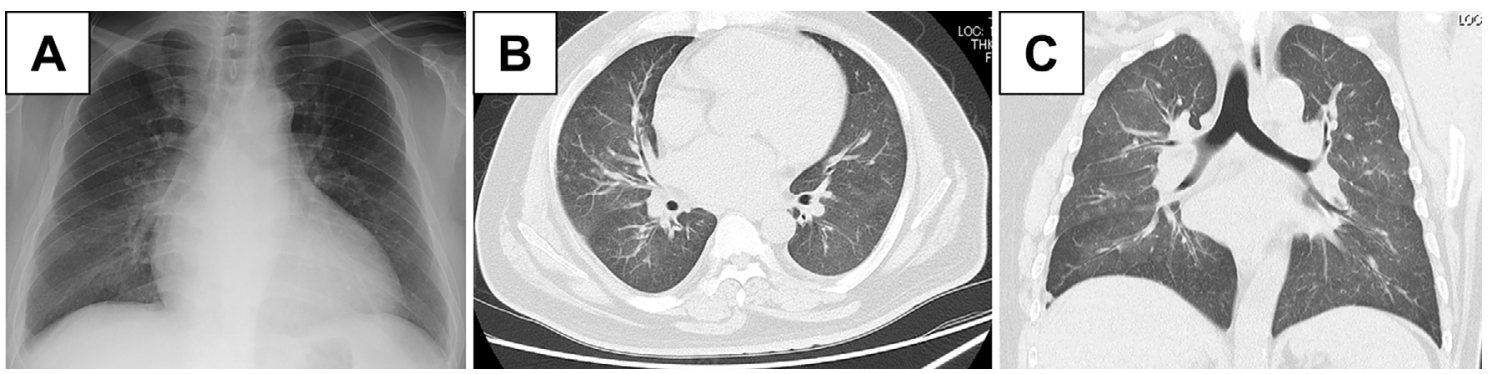

Figure 2. (A) Chest radiography showed bilateral infiltration, and (B, C) computed tomography showed marked improvement of the bilateral diffuse ground glass opacity.
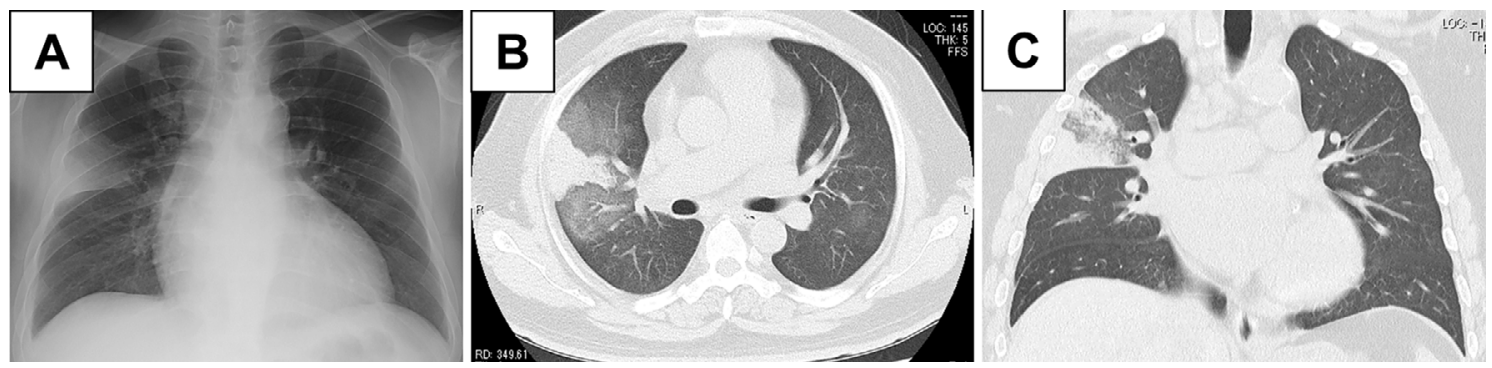

Figure 3. (A) Chest radiography and (B, C) computed tomography showed newly emerging consolidation in the right upper lobe.
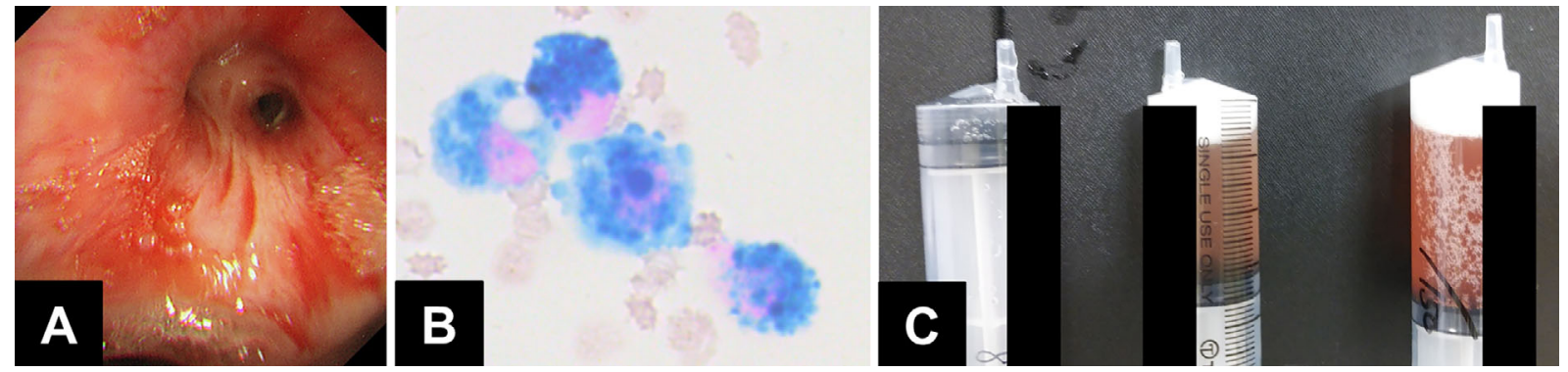

Figure 4. (A) Bronchoscopy revealed bloody effusion in the right upper bronchus. (B) A cytological examination of the bronchoalveolar lavage fluid showed hemosiderin-laden macrophages. (C) Bronchoalveolar lavage showed hemoid deposits.

to pharmacological therapy for chronic heart failure, normal imaging finding were reported with no recurrence of symptoms for 1 year.

\section{Discussion}

DAH frequently results in severe respiratory failure characterized by a high mortality and poor prognosis. In the pre- 
sent case, DAH was not fatal because of spontaneous remission. Pulmonary capillaritis in necrotizing neutrophilic interstitial pneumonitis and small-vessel vasculitis with destructive alveolar capillary basement membranes can cause DAH (8). Secondary causes of DAH involving collagen diseases, vasculitis, sarcoidosis, and amyloidosis were suspected $(9,10)$. However, combined examinations ruled out these possibilities. In addition, the patient was not taking any antiplatelet drugs or anticoagulants. Thus, we speculated that coexisting DCM and SAS might have triggered DAH via a non-immunological mechanism.

Previous reports have described combined DAH and DCM concomitant with celiac disease or idiopathic pulmonary hemosiderosis $(11,12)$, indicative of a similar underlying immunological mechanism. However, our patient did not present with gastrointestinal symptoms or hemosiderin deposits in his lungs. Further investigations were needed to detect pathogenic antibodies in order to completely rule out such possibilities.

Chronic heart failure increases pulmonary venous pressure, which might induce micro-vessel rupture, resulting in DAH $(13,14)$. Interestingly, a recent study showed that cardiovascular diseases increased long-term mortality in patients with DAH (15), which is indicative of a close pathophysiologic relationship between them.

Previous reports have also described cases of negative pressure-induced alveolar hemorrhage due to upper airway obstruction after surgery or bilateral vocal cord paralysis $(16,17)$. These findings raise the possibility that upper airway obstruction accompanied by SAS might cause and/or exacerbate alveolar hemorrhage. Thus far, there have been no reports of combined DAH and SAS, suggesting the importance of additional causative conditions.

Another report indicated that about $30 \%$ of patients with DCM-like cardiomyopathy were accompanied by obstructive sleep apnea syndrome (OSAS). Idiopathic cardiomyopathy and a high BMI in men were associated with an increased morbidity of OSAS $(18,19)$. Thus, these combined conditions might induce alveolar hemorrhage.

In summary, increased pulmonary venous pressure due to DCM and increased airway negative pressure due to SAS might have synergistically caused DAH in this case. Clinicians should note the possibility of concomitant cardiovascular diseases, especially DCM and/or SAS, in patients with DAH.

The authors state that they have no Conflict of Interest (COI).

\section{References}

1. Colby TV, Fukuoka J, Ewaskow SP, Helmers R, Leslie KO. Pathologic approach to pulmonary hemorrhage. Ann Diagn Pathol 5:
309-319, 2001

2. Lara AR, Schwarz MI. Diffuse alveolar hemorrhage. Chest 137: 1164-1171, 2010.

3. de Prost N, Parrot A, Picard C, et al. Diffuse alveolar haemorrhage: factors associated with in-hospital and long-term mortality. Eur Respir J 35: 1303-1311, 2010.

4. Martinez-Martinez MU, Oostdam DAH, Abud-Mendoza C. Diffuse alveolar hemorrhage in autoimmune diseases. Curr Rheumatol Rep 19: 27, 2017.

5. Chen XY, Sun JM, Huang XJ. Idiopathic pulmonary hemosiderosis in adults: review of cases reported in the latest 15 years. Clin Respir J 11: 677-681, 2017.

6. Ioachimescu OC, Sieber S, Kotch A. Idiopathic pulmonary haemosiderosis revisited. Eur Respir J 24: 162-170, 2004.

7. Sherani KM, Upadhyay HN, Sherani FK, Vakil AP, Sarkar SS. Idiopathic pulmonary hemosiderosis presenting in an adult: a case report and review of the literature. Lung India 32: 395-397, 2015.

8. Mark EJ, Ramirez JF. Pulmonary capillaritis and hemorrhage in patients with systemic vasculitis. Arch Pathol Lab Med 109: 413418, 1985.

9. Tajima S, Hironaka M, Nakazawa S, et al. Alveolar hemorrhage in a patient with sarcoidosis. Sarcoidosis Vasc Diffuse Lung Dis 23: 236-237, 2006.

10. Shenin M, Xiong W, Naik M, Sandorfi N. Primary amyloidosis causing diffuse alveolar hemorrhage. J Clin Rheumatol 16: 175177, 2010.

11. Isikay S, Yilmaz K, Kilinc M. Celiac disease with pulmonary haemosiderosis and cardiomyopathy. BMJ Case Rep 2012: bcr 2012007262, 2012.

12. Khilnani GC, Jain N, Tiwari P, Hadda V, Singh L. A young man with hemoptysis: rare association of idiopathic pulmonary hemosiderosis, celiac disease and dilated cardiomyopathy. Lung India 32: 70-72, 2015.

13. Jonckheer J, Slabbynck H, Spapen H. Diffuse alveolar haemorrhage with predominant upper lung lobe involvement associated with congestive heart failure: a case series. J Thorac Dis 9: 39563960, 2017.

14. Suzuki A, Nakagawa N, Maruyama K, Matsuki M, Hasebe N. Diffuse alveolar hemorrhaging with hypertensive emergency: A Rare but Important Cause of Hemoptysis. Intern Med 58: 1511-1516, 2019.

15. Tamai K, Tomii K, Nakagawa A, Otsuka K, Nagata K. Diffuse alveolar hemorrhage with predominantly right-sided infiltration resulting from cardiac comorbidities. Intern Med 54: 319-324, 2015.

16. Contou D, Voiriot G, Djibre M, Labbe V, Fartoukh M, Parrot A. Clinical features of patients with diffuse alveolar hemorrhage due to negative-pressure pulmonary edema. Lung 195: 477-487, 2017.

17. Schwartz DR, Maroo A, Malhotra A, Kesselman H. Negative pressure pulmonary hemorrhage. Chest 115: 1194-1197, 1999.

18. Sin DD, Fitzgerald F, Parker JD, Newton G, Floras JS, Bradley TD. Risk factors for central and obstructive sleep apnea in 450 men and women with congestive heart failure. Am J Respir Crit Care Med 160: 1101-1106, 1999.

19. Banno K, Shiomi T, Sasanabe R, et al. Sleep-disordered breathing in patients with idiopathic cardiomyopathy. Circ J 68: 338-342, 2004.

The Internal Medicine is an Open Access journal distributed under the Creative Commons Attribution-NonCommercial-NoDerivatives 4.0 International License. To view the details of this license, please visit (https://creativecommons.org/licenses/ by-nc-nd/4.0/).

(C) 2021 The Japanese Society of Internal Medicine

Intern Med 60: 1911-1914, 2021 